\title{
Wavelength-spacing tunable S-band multi-wavelength fiber laser based on Lyot filter
}

\begin{abstract}
We demonstrated a tunable S-band multiwavelength fiber laser based on semiconductor optical amplifier (SOA) and hybrid amplifier. The proposed multiwavelength utilizes a comb filter based on one PMF segment of Lyot birefringence filter for multiwavelength tunability. The wavelength spacing and the peak flatness can be altered by adjusting polarization controller (PC). The wavelength spacing tunability is either $0.4 \mathrm{~nm}$ or $0.8 \mathrm{~nm}$ with a little peak fluctuations in S-band waveband region. We obtained 6 and 10 lasing wavelengths within $10 \mathrm{~dB}$ bandwidth at wavelength spacing of $0.8 \mathrm{~nm}$ and $0.4 \mathrm{~nm}$, respectively. EDFA is added into the structure and the result shows that the extinction ratio (ER) is increased to more than $30 \mathrm{~dB}$ which is due to higher gain provided.
\end{abstract}

